\title{
A note on the singular chromatic number of some product graphs
}

\author{
N. Paramaguru ${ }^{1,2}$
}

\begin{abstract}
A proper coloring of a graph $G$ is an assignment of colors to the vertices of $G$ such that adjacent vertices are assigned distinct colors. The minimum number of colors in a proper coloring of $G$ is the chromatic number $\chi(G)$ of $G$. For a graph $G$ and a proper coloring $c: V(G) \rightarrow\{1,2, \ldots, k\}$ of the vertices of $G$ for some positive integer $k$, the color code of a vertex $v$ of $G$ (with respect to $c$ ) is the ordered pair code $(v)=\left(c(v), S_{v}\right)$, where $S_{v}=\{c(u): u \in N(v)\}$. The coloring $c$ is singular if distinct vertices have distinct color codes and the singular chromatic number $\chi_{s i}(G)$ of $G$ is the minimum positive integer $k$ for which $G$ has a singular $k$-coloring. In this paper, we determine the singular chromatic number of Cartesian product of paths.
\end{abstract}

\section{Keywords}

Proper coloring, chromatic number, singular chromatic number.

\section{AMS Subject Classification} 05C15, 05C22.

${ }^{1}$ Department of Mathematics, Government Arts College for Women, Krishnagiri-635002, Tamil Nadu, India.

${ }^{2}$ Department of Mathematics, Annamalai University, Annamalai Nagar-608002, Tamil Nadu, India.

Corresponding author: npguru@gmail.com

Article History: Received 24 March 2019; Accepted 09 May 2019

(C)2019 MJM.

\section{Contents}

1 Introduction 151

2

References 151 153

\section{Introduction}

For a graph $G$, let $c: V(G) \rightarrow \mathbb{N}$ be a proper vertex coloring of $G$. For each vertex $v$ of $G$, let $S_{v}$ be the set of colors of the neighbors of $v$, that is, $S_{v}=\{c(u): u \in N(v)\}$ where $N(v)$ is the neighborhood of $v$. The color code code $(v)$ of $v$ is then defined as the ordered pair $\left(c(v), S_{v}\right)$. If $\operatorname{code}(u) \neq \operatorname{code}(v)$ for every two distinct vertices $u$ and $v$ of $G$, then $c$ is called a $\sin$ gular coloring of $G$. Therefore, a singular coloring of a graph $G$ is a coloring that uses the color of each vertex together with the set of colors of its neighbors to distinguish all vertices in $G$. If a singular coloring $c$ uses $k$ colors, then $c$ is a singular $k$-coloring. For each positive integer $k$, let $\mathbb{N}_{k}=\{1,2, \ldots, k\}$. Thus, we assume that every singular $k$-coloring uses the colors in $\mathbb{N}_{k}$. A graph $G$ is singularly $k$-colorable if $G$ has a singular $k$-coloring.

The minimum $k$ for which $G$ has a singular $k$-coloring is called the singular chromatic number of $G$ and is denoted by $\chi_{s i}(G)$. Since a coloring assigning distinct colors to distinct vertices of a graph $G$ is a singular coloring of $G$, the singular chromatic number exists for every graph. The concept of singular coloring was introduced by Kolasinski, Lin and Okamoto [3].

In [3], Kolasinski, Lin and Okamoto characterized: Let $G$ be a graph of order $n$, then $\chi_{s i}(G)=n$ if and only if $G$ is a complete multipartite graph or an empty graph; and also proved that: For each $n \geq 3$, let $k$ be the unique positive integer such that $(k-1)\left(\begin{array}{c}k-1 \\ 2\end{array}\right)+1 \leq n \leq k\left(\begin{array}{c}k \\ 2\end{array}\right)$, then $\chi_{s i}\left(P_{n}\right)=$ $k, \chi_{s i}\left(C_{n}\right)=k$ if $n \neq k\left(\begin{array}{l}k \\ 2\end{array}\right)-1 ; \chi_{s i}\left(C_{n}\right)=k+1$ if $n=k\left(\begin{array}{l}k \\ 2\end{array}\right)-1$.

The Cartesian product $G \square H$ of two graphs $G$ and $H$ has $V(G \square H)=V(G) \times V(H)$, and two vertices $\left(u_{1}, u_{2}\right)$ and $\left(v_{1}, v_{2}\right)$ of $G \square H$ are adjacent if and only if either $u_{1}=v_{1}$ and $u_{2} v_{2} \in E(H)$ or $u_{2}=v_{2}$ and $u_{1} v_{1} \in E(G)$.

In this paper, we compute the singular chromatic number of Cartesian product of paths.

\section{Results}

We have the following observations:

Observation 2.1. [3] For every graph $G$ of order $n, \chi(G) \leq$ $\chi_{s i}(G) \leq n$. 
Observation 2.2. [3] Let $G$ be a graph.

(a) $\chi_{s i}(G)=1$ if and only if $G=K_{1}$.

(b) $\chi_{s i}(G)=2$ if and only if $G=\left\{K_{2}, K_{2} \cup K_{1}, K_{2} \cup 2 K_{1} \cdot\right\}$

Observation 2.3. [3] If $\chi_{s i}(G)=k$, then each of the following holds.

(a) $G$ contains at most $k$ isolated vertices.

(b) $G$ contains at most one clique of order $k$.

(c) Each vertex in $G$ is adjacent to at most $k-1$ end-vertices.

For the path $P_{n}$ on $n$ vertices, let $V\left(P_{n}\right)=\{1,2, \ldots, n\}$, $E\left(P_{n}\right)=\{\{i, i+1\}: i \in\{1,2, \ldots, n-1\}\}$.

Let $c((i, j))$ denotes the color assigned to the vertex $\left(v_{i}, v_{j}\right)$ and code $((i, j))$ denotes the color code of the vertex $\left(v_{i}, v_{j}\right)$.

The following figures shows that the singular colorings of $P_{3} \square P_{3}, P_{3} \square P_{4}$ and $P_{3} \square P_{5}$.

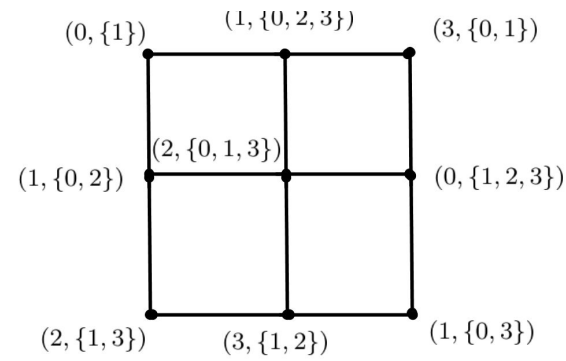

Figure 1. The singular 4-coloring of $P_{3} \square P_{3}$

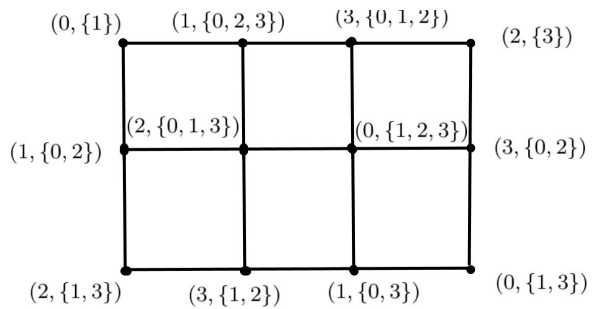

Figure 2. The singular 4-coloring of $P_{3} \square P_{4}$

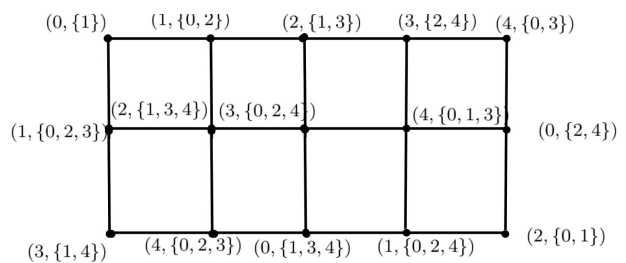

Figure 3. The singular 5-coloring of $P_{3} \square P_{5}$

Theorem 2.4. For $n \geq 6, \chi_{s i}\left(P_{3} \square P_{n}\right) \leq n-1$.

Proof. Define $c: V\left(P_{3} \square P_{n}\right) \rightarrow\{0,1,2, \ldots, n-2\}$ as follows: $c((1, j)) \equiv(j-1)(\bmod (n-1)), c((2, j)) \equiv j(\bmod (n-$ $1))$ and $c((3, j)) \equiv(j+2)(\bmod (n-1))$, where $j \in\{1,2,3, \ldots$,

Then, code $((1,1))=(0,\{1\})$; $\operatorname{code}((1, j))=((j-1)(\bmod (n-1)),\{(j-2)(\bmod (n-1)), j($ $\bmod (n-1))\})$ if $j \in\{2,3,4, \ldots, n\}$;

$\operatorname{code}((2, j))=(j(\bmod (n-1)),\{(j-1)(\bmod (n-1)),(j+$ 1) $(\bmod (n-1)),(j+2)(\bmod (n-1))\})$ if $j \in\{1,2,3, \ldots, n-$ $1\}$;

$\operatorname{code}((2, n))=(1,\{0,3\})$

$\operatorname{code}((3, j))=((j+2)(\bmod (n-1)),\{j(\bmod (n-1)),(j+$

$1)(\bmod (n-1)),(j+3)(\bmod (n-1))\})$ if $j \in\{2,3,4, \ldots, n-$ $1\}$;

$\operatorname{code}((3,1))=(3,\{1,4\})$;

code $((3, n))=(3,\{1,2\})$. Hence, $\chi_{s i}\left(P_{3} \square P_{n}\right) \leq n-1$.

Theorem 2.5. For $m \geq 3, n \geq 3$, and $m \leq n, \chi_{s i}\left(P_{m} \square P_{n}\right) \leq$ $n+1$.

Proof.

Let us define $c: V\left(P_{m} \square P_{n}\right) \rightarrow\{0,1,2, \ldots, n\}$ as follows:

$c((i, j))=j-1$ if $(i, j) \in(\{1\} \times\{1,2,3, \ldots, n\})$;

$c((i, j))=j$ if $(i, j) \in(\{2\} \times\{1,2,3, \ldots, n\})$;

$c((i, j))=(i+j-1) \equiv 0 \bmod (n+1)$ if $(i, j) \in(\{3,7,11, \ldots\} \times$

$\{1,2,3, \ldots, n\})$;

$c((i, j))=(i+j) \equiv 0 \bmod (n+1)$ if $(i, j) \in(\{4,8,12, \ldots\} \times$

$\{1,2,3, \ldots, n\})$;

$c((i, j))=\left(\left\lfloor\frac{i}{2}\right\rfloor+j-1\right) \equiv 0 \bmod (n+1)$ if $(i, j) \in(\{5,9,13, \ldots\} \times$

$\{1,2,3, \ldots, n\})$

$c((i, j))=\left(\frac{i}{2}+j\right) \equiv 0 \bmod (n+1)$ if $(i, j) \in(\{6,10,14, \ldots\} \times$

$\{1,2,3, \ldots, n\})$

then the color code is:

code $((1,1))=(0,\{1\})$;

code $((i, j))=(j-1,\{j-2, j\})$ if $(i, j) \in(\{1\} \times\{2,3, \ldots, n\})$;

$\operatorname{code}((i, j))=(j,\{j-1, j+1,(j+2) \equiv 0 \bmod (n+1)\})$ if

$(i, j) \in(\{2\} \times\{1,2,3, \ldots, n-1\})$;

$\operatorname{code}((2, n))=(n,\{1, n-1\})$;

$\operatorname{code}((i, j))=(j+2 \equiv 0 \bmod (n+1),\{j \equiv 0 \bmod (n+$

$1),(j+3) \equiv 0 \bmod (n+1),(j+4) \equiv 0 \bmod (n+1),(j+$

$1) \equiv 0 \bmod (n+1)\})$ if $(i, j) \in(\{3\} \times\{2,3,4, \ldots, n-1\})$;

$\operatorname{code}((3,1))=(3,\{1,4,5\})$;

$\operatorname{code}((3, n))=(1,\{0,3, n\})$;

$\operatorname{code}((i, j))=((i+j) \equiv 0 \bmod (n+1), \quad\{(i+j-2) \equiv 0$ $\bmod (n+1),(i+j+1) \equiv 0 \bmod (n+1),\left(\frac{i}{2}+j-1\right) \equiv$ $0 \bmod (n+1), \quad(i+j-1) \equiv 0 \bmod (n+1)\})$ if $(i, j) \in$ $(\{4,8,12, \ldots, \neq m\} \times\{2,3,4, \ldots, n-1\}) ;$

$\operatorname{code}((i, j))=((i+j) \equiv 0 \bmod (n+1), \quad\{(i+j-2) \equiv 0$ $\left.\left.\bmod (i+j+1) \equiv 0 \bmod (n+1),\left(\frac{i}{2}\right) \equiv 0 \bmod (n+1)\right\}\right)$ if $(i, j) \in(\{4,8,12, \cdots \neq m\} \times\{1\})$;

$\operatorname{code}((i, j))=\left((i-1) \equiv 0 \bmod (n+1),\left\{(i-3) \equiv 0 \bmod \left(\frac{i}{2}-\right.\right.\right.$ $2) \equiv 0 \bmod (n+1),(i-2) \equiv 0 \bmod (n+1)\})$ if $(i, j) \in$ $(\{4,8,12, \cdots \neq m\} \times\{n\})$ $\operatorname{code}((i, j))=\left(\left(\left\lfloor\frac{i}{2}\right\rfloor+j-1\right) \equiv 0 \bmod (n+1), \quad\{(i+j-\right.$ $1) \equiv 0 \bmod (n+1),\left(\left\lfloor\frac{i}{2}\right\rfloor+j-2\right) \equiv 0 \bmod (n+1),\left(\left\lfloor\frac{i}{2}\right\rfloor+\right.$ $\left.\left.j) \equiv 0 \bmod (n+1),\left(\left\lfloor\frac{i}{2}\right\rfloor+j+1\right) \equiv 0 \bmod (n+1)\right\}\right)$ if $(i, j) \in(\{5,9,13, \cdots \neq m\} \times\{2,3,4, \ldots, n-1\})$;

$\operatorname{code}((i, j))=\left(\left(\left\lfloor\frac{i}{2}\right\rfloor\right) \equiv 0 \bmod (n+1),\{(i) \equiv 0 \bmod (n+\right.$ $1),\left(\left\lfloor\frac{i}{2}\right\rfloor+j\right) \equiv 0 \bmod (n+1),\left(\left\lfloor\frac{i}{2}\right\rfloor+j+1\right) \equiv 0 \bmod (n+$ $n\})\}$.$) if (i, j) \in(\{5,9,13, \ldots\} \times\{1\})$;

$\operatorname{code}((i, j))=\left(\left(\left\lfloor\frac{i}{2}\right\rfloor+j-1\right) \equiv 0 \bmod (n+1), \quad\{(i+j-\right.$ 
1) $\equiv 0 \bmod (n+1),\left(\left\lfloor\frac{i}{2}\right\rfloor+j+1\right) \equiv 0 \bmod (n+1),\left(\left\lfloor\frac{i}{2}\right\rfloor+\right.$ $j-2) \equiv 0 \bmod (n+1)\}$,$) if (i, j) \in(\{5,9,13, \cdots \neq m\} \times$ $\{n\})$;

code $((i, j))=\left(\left(\frac{i}{2}+j\right) \equiv 0 \bmod (n+1), \quad\left\{\left(\frac{i}{2}+j-2\right) \equiv 0\right.\right.$

$\bmod (n+1), \quad\left(\frac{i}{2}+j+1\right) \equiv 0 \bmod (n+1), \quad(i+j) \equiv 0$

$\left.\left.\bmod (n+1), \quad\left(\frac{i}{2}+j-1\right) \equiv 0 \bmod (n+1),\right\}\right)$ if $(i, j) \in$ $(\{6,10,14, \cdots \neq m\} \times\{2,3,4, \ldots, n-1\}) ;$

$\operatorname{code}((i, j))=\left(\left(\frac{i}{2}+j\right) \equiv 0 \bmod (n+1),\left\{\left(\frac{i}{2}+j-2\right) \equiv 0\right.\right.$

$\bmod (n+1), \quad\left(\frac{i}{2}+j+1\right) \equiv 0 \bmod (n+1), \quad(i+j) \equiv 0$

$\bmod (n+1)\}$,$) if (i, j) \in(\{6,10,14, \cdots \neq m\} \times\{1\})$;

$\operatorname{code}((i, j))=\left(\left(\frac{i}{2}+j\right) \equiv 0 \bmod (n+1),\left\{\left(\frac{i}{2}+j-2\right) \equiv 0\right.\right.$

$\bmod (n+1), \quad\left(\frac{i}{2}+j-1\right) \equiv 0 \bmod (n+1), \quad(i+j) \equiv 0$

$\bmod (n+1)\}$,$) if (i, j) \in(\{6,10,14, \cdots \neq m\} \times\{1\})$;

$\operatorname{code}((i, j))=\left((i+j-1) \equiv 0 \bmod (n+1),\left\{\left(\left\lfloor\frac{i}{2}\right\rfloor+j\right) \equiv\right.\right.$

$0 \bmod (n+1), \quad(i+j) \equiv 0 \bmod (n+1), \quad(i+j+1) \equiv$

$0 \bmod (n+1), \quad(i+j-2) \equiv 0 \bmod (n+1)\})$ if $(i, j) \in$

$(\{7,11,15 \cdots \neq m\} \times\{2,3,4, \ldots, n-1\})$;

$\operatorname{code}((i, j))=\left(i,\left\{\left\lfloor\frac{i}{2}\right\rfloor+1, i+1, i+2\right\}\right)$ if $(i, j) \in(\{7,11,15, \cdots \neq$

$m\} \times\{1\})$;

$\operatorname{code}((i, j))=\left((i+j-1) \equiv 0 \bmod (n+1),\left\{\left(\left\lfloor\frac{i}{2}\right\rfloor+j\right) \equiv 0\right.\right.$

$\bmod (n+1),(i+j-2) \equiv 0 \bmod (n+1),(i+j+1) \equiv 0$

$\bmod (n+1)\})$ if $(i, j) \in(\{7,11,15, \cdots \neq m\} \times\{n\})$;

Finally, we have

Case(1). $m \equiv 0 \bmod 4$.

$\operatorname{code}((m, 1))=(0,\{1, m-1\})$;

code $((m, n))=(n-1,\{n-3, n-2\})$;

$\operatorname{code}((i, j))=(j-1, \quad\{(j-2) \equiv 0 \bmod (n+1), \quad j \equiv 0$

$\bmod (n+1),(i+j-2) \equiv 0 \bmod (n+1)\})$ if $(i, j) \in(\{m\} \times$

$\{2,3, \ldots, n-1\})$

Case (2). $m \equiv 1 \bmod 4$.

$\operatorname{code}((m, 1))=\left(\left\lfloor\frac{m}{2}\right\rfloor,\left\{n,\left\lfloor\frac{m}{2}\right\rfloor+1\right\}\right) ;$

code $((m, n))=\left(\left[\frac{m}{2}\right]-2,\left\{\left[\frac{m}{2}\right]-1, n-2\right\}\right)$;

$\operatorname{code}((i, j))=\left(\left(\left\lfloor\frac{i}{2}\right\rfloor+j-1\right) \equiv 0 \bmod (n+1),\{(m+j-\right.$ $1) \equiv 0 \bmod (n+1), \quad\left(\left\lfloor\frac{i}{2}\right\rfloor+j \equiv 0 \bmod (n+1), \quad\left(\left\lfloor\frac{i}{2}\right\rfloor+\right.\right.$ $j-2) \equiv 0 \bmod (n+1)\})$ if $(i, j) \in(\{m\} \times\{2,3, \ldots, n-$ $1\})$;

Case(3). $m \equiv 2 \bmod 4$.

$\operatorname{code}((m, 1))=\left(\frac{m}{2}+1,\left\{\frac{m}{2}-1, \frac{m}{2}+2\right\}\right) ;$

$\operatorname{code}((m, n))=\left(\frac{m}{2}-1,\left\{\frac{m}{2}-3, \frac{m}{2}-2\right\}\right)$

$\operatorname{code}((i, j))=\left(\left(\frac{m}{2}+j\right) \equiv 0 \bmod (n+1),\left\{\left(\frac{m}{2}+j-2\right) \equiv 0\right.\right.$

$\left.\bmod (n+1),\left(\frac{m}{2}+j+1\right) \equiv 0 \bmod (n+1), \frac{m}{2}+j-1\right) \equiv 0$

$\bmod (n+1)\})$ if $(i, j) \in(\{m\} \times\{2,3, \ldots, n-1\})$;

Case(4). $m \equiv 3 \bmod 4$.

$\operatorname{code}((m, 1))=\left(m,\left\{\left\lfloor\frac{m}{2}\right\rfloor+1,(m+1) \equiv 0 \bmod (n+1)\right\}\right) ;$ $\operatorname{code}((m, n))=\left(m-2,\left\{\left\lfloor\frac{m}{2}\right\rfloor-1, m-3\right\}\right)$;

$\operatorname{code}((i, j))=\left((m+j-1) \equiv 0 \bmod (n+1),\left\{\left(\left\lfloor\frac{m}{2}\right\rfloor+j\right) \equiv\right.\right.$ $0 \bmod (n+1),(m+j) \equiv 0 \bmod (n+1),(m+j-2) \equiv 0$ $\bmod (n+1)\})$ if $(i, j) \in(\{m\} \times\{2,3, \ldots, n-1\})$.

Hence, $\chi_{s i}\left(P_{m} \square P_{n}\right) \leq n+1$.

\section{References}

[1] R. Balakrishnan and K. Ranganathan, A Textbook of Graph Theory, Springer-Verlag, New York, 2000.

[2] G. Chartrand and P. Zhang, Chromatic Graph Theory, Chapman Hall/CRC press, Boca Raton (2009).

[3] K. Kolasinski, J. Lin and F. Okamoto, The singular Chromatic Number of a Graph, Ars Combinatoria, 118 (2015) 13-31.
$\operatorname{ISSN}(\mathrm{P}): 2319-3786$

Malaya Journal of Matematik

$\operatorname{ISSN}(\mathrm{O}): 2321-5666$

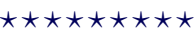

